\title{
Reflections on the First Bi-annual Endicott College Expressive Therapies Symposium: Traditional Chinese Medicine, Arts, and Culture
}

\author{
关于第一届两年一度的恩迪科特大学表达疗法研讨会的思考 : \\ 中医，艺术与文化 \\ Krystal Demaine \\ Endicott College, USA
}

\begin{abstract}
This article shares a reflection of the first bi-annual Endicott College Expressive Therapies Symposium: Traditional Chinese Medicine, Arts, and Culture. The purpose of the symposium was to maintain a collaborative and cross-cultural dialog between traditional Chinese medicine (TCM) practitioners and Western creative arts therapists.
\end{abstract}

Keywords: Expressive Therapies Symposium, Traditional Chinese Medicine, Chinese Culture, Arts, Guqin, Daoism

\section{摘要}

本文分享了第一届两年一度的Endicott大学表达疗法专题讨论会 : 传统中医药，艺术与 文化。研讨会目的是持续传统中医 (TCM ) 从业者与西方创造艺术治疗师之间的协作 及跨文化对话。

关键字 : 表达疗法研讨会，中医，中国文化，艺术，古琴，道

\section{Introduction}

In the fall of 2018, I set forth on a mission to bring a dynamic one-day symposium experience to Endicott College in Beverly, MA, USA. After I previously collaborated with Chunhong Wang and Dr. Jane Ferris Richardson, during an Endicott Collegehosted workshop in 2016 (Demaine, 2016), as well as prior projects in Beijing, China (Demaine, 2015; Demaine \& Richardson, 2015); I decided to invite both collaborators to consult with me on a theme for what would be the first Endicott College Expressive Therapies Symposium. Wang and Ferris-Richardson have been my collaborators since we worked together on the Creative Arts and Natural Health Conference in Beijing China in 2009. For the very first event, the focus would be on Eastern and Western perspectives, with specific attention on China. It was decided that the topic title, Traditional Chinese Medicine, Arts, and Culture, would allow the symposium to highlight the deeply artistic, and very ancient culture of China within multiple art forms. 
The event attracted 70 participants from the New England Region and beyond, reaching as far as Atlanta, GA, USA. This symposium was made possible in part by sponsors who included Endicott College, The Van Otterloo Family Foundation, and the International Expressive Arts Therapy Association (IEATA).

\section{Creating an East-West Dialog}

"In China, we learned the healers were artists, and the artists were healers"

(Demaine \& Ferris-Richardson, 2015, p. 59).

In order to create an opportunity for unique voices to be heard at the symposium, I invited presenters from multiple arts disciplines; who had trained as traditional Chinese medicine (TCM) doctors, or whom have studied the use of Chinese medicine within the context of the expressive and creative arts therapies. I had previously written about the creative (musical) roots of healing within the context of Chinese medicine and indigenous cultures (Demaine, 2015, 2016; Demaine \& Ferris-Richardson, 2015) and had a strong sense that the information that I have learned from my experiences would be worth engaging with in a deeper dialog.

In the West allopathic medicine is key; with the goal to treat disease using drug medicines or surgical interventions. For thousands of years prior to the advent of this Western medicine, traditional medicines such as those found in sacred and cultural practices were used by traditional healers; including yoga, meditation, acupuncture, massage, or tai chi, which in the West are called complimentary or alternative approaches to healing. The merging of these two approaches is called integrative care - the collaboration between the East and the West and an integration of indigenous and European traditions.

We understand that the body holds trauma, and we naturally draw upon movement to express and work through the pain of trauma. We understand the importance of restoring the natural sleep-wake cycle, and the role of stress, inflammation, leading to depression and pain, then exacerbated by leading to self-medication.

It is well documented that medicine has been of central importance to Chinese civilization (Reid, 1996). The TCM system is one that is grounded in energy and the functions of the body, which are housed in the polarities of Yin and Yang. It was the Yellow Emperor's Classic of Internal Medicine, the so-called manual for the study of traditional Chinse medicine, that evolved the theory of the five elements, five energies, or five organs - which relate to all the human body's systems. These elements: fire, water, metal, wood, and earth are enigmatically connected to five emotions, five, colors, five musical tones, five sensory experiences, and so forth (Demaine, 2015). Treatment in TCM revolves around the expertise of a traditional Chinese medicine doctor who may specialize in one or more areas of treatment, such as acupuncture, tai chi, or medicinal use of Chinese herbal products, but has a general knowledge of the use of how the treatment modalities interact within the five element theory of TCM.

Knowing the relevance, and roots of connection for the role of TCM within the field of expressive therapies is the reason I chose to host an event steeped in this theme. The 
purpose of the symposium was to engage in a discussion and create a dialog between two different fields that merge sometimes unknowingly in parallel with holistic treatment for an individual or group. As the roots of traditional healing approaches are grounded in the arts, this led how the Endicott College Expressive Therapies Symposium evolved over the day; through the integration of invited presenters who were skillfully trained in the areas of visual arts, music, and movement through printmaking, drumming, tai chi, assemblage, traditional Chinese paper cutting, music performance, and creative movement.

\section{Opening Remarks and Keynote}

\section{"Values of working for the benefit of community are deeply rooted throughout East Asia" (Shaun McNiff)}

The morning schedule began in the Rose Auditorium in the School of Visual and Performing Arts at Endicott College. The opening remarks by Dean Mark Towner, symposium consultant Chunhong Wang, and symposium organizer Dr. Krystal Demaine were instrumental in setting the intentions for the day. Dean Towner shared the impassioned stories of how art healed him over the course of his life, and a brief history of the expressive arts programs at Endicott College, while Wang and Dr. Demaine introduced the mission of the symposium as well as the schedule for the day.

The mission...

To cultivate a dynamic and creative conversation within the relationships and intersections between the expressive therapies and traditional Chinese medicine, arts, healing, and culture, and to support diversity within the expressive arts communities.

The opening remarks were followed by a digital presentation by Dr. Tony Zhou, Executive Editor and Founder of Creative Arts in Education and Therapy (CAET). Dr. Zhou's talk underscored the mission of CAET in furthering the cooperation and understanding between China and the West. Dr. Zhou's opening was followed by the keynote presentation by his Western counterpart, Co-Editor-in-Chief of CAET, Shaun McNiff. His talk, "East West Convergence on How Art Heals: China's Many Contributions", set the stage for the conference dialog between China and the West. The presentation focused on "what we can learn from China regarding how art heals as contrasted to the worldwide tendency to adopt Western conventions." Conference participants appreciated the emphasis on "shared humanity and trans-cultural understanding". This was demonstrated by a showing of the art made by untrained artists in the Chinese and Japanese studio workshops featured in issues 4.1 and 5.1 of CAET (links) together with art featured in the Art Therapy at Danvers exhibition (https://www.danversstatehospital.org/art-therapy) in the early 1970s which did so much to launch the arts in therapy field while furthering public understanding of the human dignity of the artists as also emphasized by the workshops featured in the journal. 


\section{Break Out Sessions}

"We are growing together in order to create a global bridge of healing, understanding, and widening the East and West creative bridge" (Demaine, 2016, p. 38).

At the conclusion of the opening talks, participants separated into three different preregistered groups in order to attend a small 75-minute group workshop. The sessions were conducted in three different studio spaces, offering intimate engagement in visual and performing arts. Chunhong Wang, a traditional Chinese medicine practitioner and founder of Dimensional Arts Dance Therapy, of Beijing China, offered a workshop in the Black Box Dance Studio titled "Yin Yang Energy: The Color and Shape of Working with Diagnosis". Wang's workshop invited participants to examine the role of color, movement, intention, and partnerships within the context of yin and yang energy. Participants received colorful paper and a pair of scissors and were then instructed in basic Chinese paper cut form (see Figure 1). With a partner each discussed the paper cut they had created, the color of the paper chosen, and the relationship between the

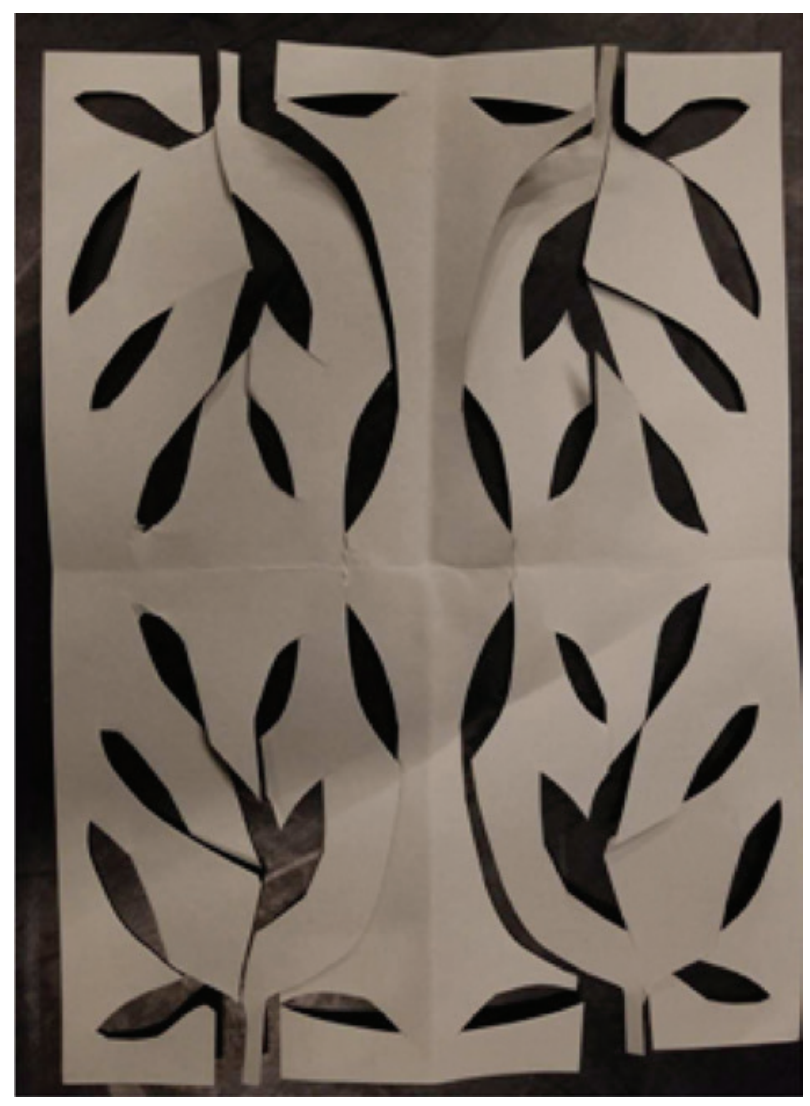

FIGURE 1 | A basic Chinese paper cut. 
partnership to examine yin and yang philosophy which draws upon a mutual relationship of restraint, transformation of inner emotion and outside action working in balance. Partners discussed the stillness of yin and the activity of yang that was demonstrated it the cuts in the paper. Each partnership found flow between the yin and yang of the activity presented on the paper with a final movement performance. Wang's method of yin-yang is working toward diagnosis and understanding of human emotions and relationships.

Meanwhile, in the Creative Arts Studio, expressive arts therapist, Dr. Mitchell Kossak, of Cambridge, MA, USA, offered the workshop titled, "Five Elements and Expressive Arts: An Ayureda Perspective". Kossak's workshop offered an integration of a lecture, movement, drumming, reflective art making, partner discussion, and group sharing. His lecture was grounded in his experiences and personal studies in China on the use of arts and the five-element healing system. Kossak's work on therapeutic attunement in the expressive arts (2015) provides the framework for his engagement embodied empathetic resonance that he lectures on and practices with a multi-art approach involving sound, rhythm, art making, and movement.

The third break out session, in the print making studio was facilitated by art therapist/play therapist Dr. Jane Ferris Richardson and her son, printmaker, Gabriel Richardson, of New Bedford, MA, USA. Together, they collaborated to offer a workshop titled "Art as a Heart Print: Chinese Painting Traditions and Contemporary Monotype for Art Therapy Practices". Using nature as a guide, Ferris-Richardson noted that Chinese artists work to evoke feelings of movement and flow based on the

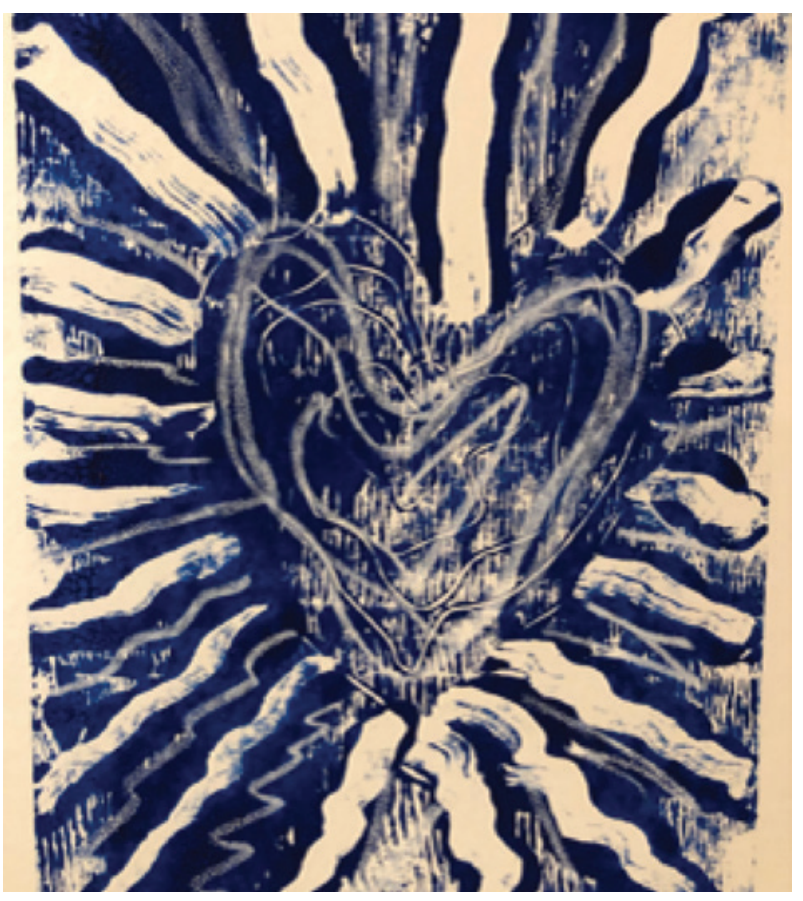

FIGURE 2 | Printmaking heart. 
deep connection to nature, and that in Chinese painting there is a relationship between the artists and the painted world, and that this art allows the artist to be truly seen, with authenticity and resonance. Participants in this workshop were introduced to a brief lecture on authentic art making, and monoprint techniques, to be used as a rich fine art process in art therapy. At the end of the workshop each participant shared a print they had created. For one participant a heart emerged (see Figure 2.) through the process facilitated in the workshop.

The workshop choices were intended to offer diverse experiences in the integration of the arts with consideration of Chinese arts and culture. Each of the presenters engaged in their small group experiences of no more than 25 participants in each group in order to provide thoughtful and meaningful art making, discussion, and collaboration.

In the atrium, participants found the five-element mandala, the portable altars, and a live performance, all of which could be explored independently. The five-element mandala was designed by Demaine and Wang and composed of dried beans with corresponding colors to the five-element theory (red, green, yellow, white, and black). The beans were fanned on a table where participants could engage in a flow of design as they walked by. The integration of colors showed how the elements interact with one another and rely on each other for (flow) ch'i. Figure 3 shows the natural engagement that occurred with the bean mandala as people walked by to interact with the objects.

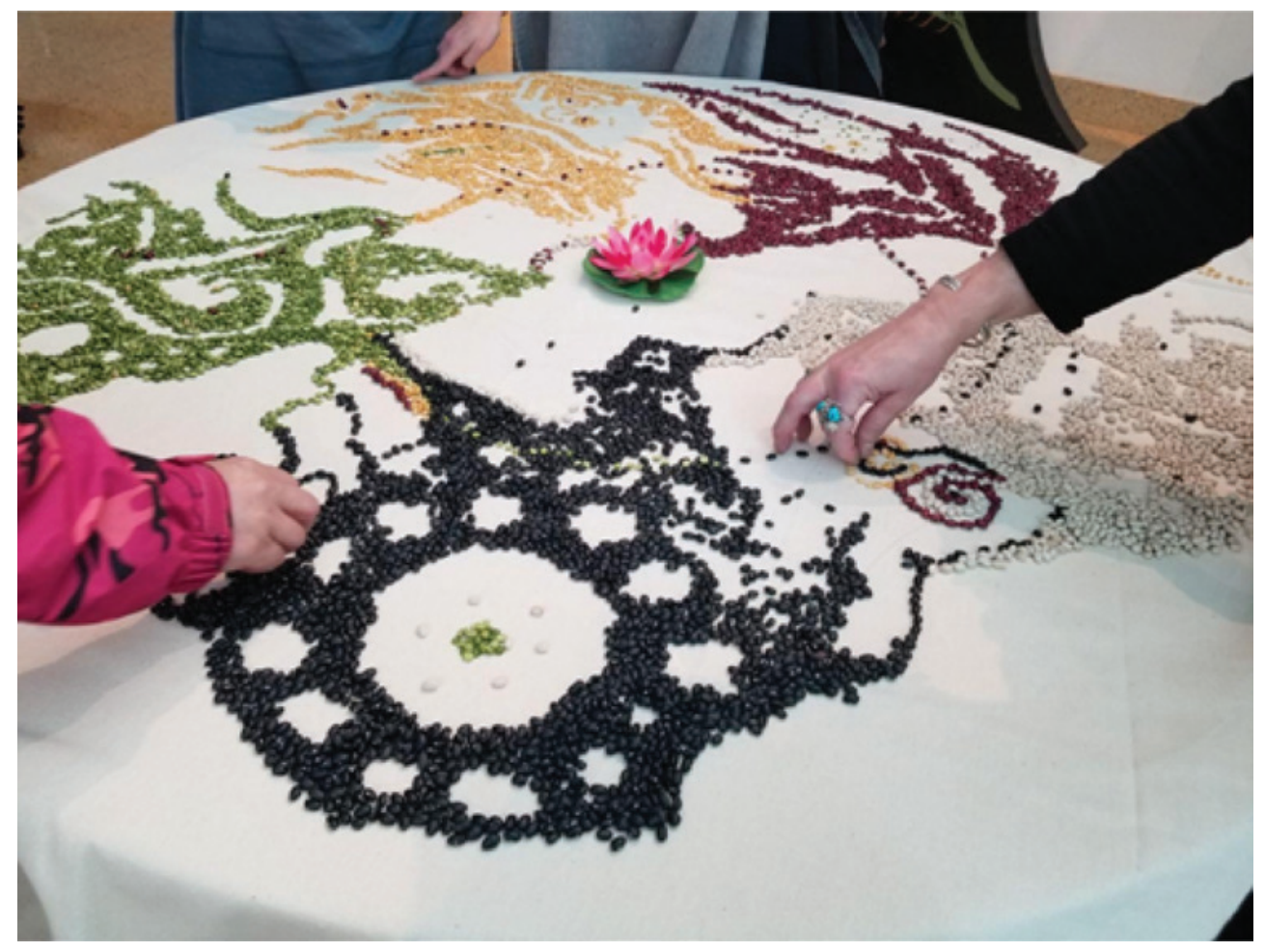

FIGURE 3 | Five element mandala. 
The open art activity cultivated by New York-based Chinese Artist, Tattfoo Tan, known as portable altars allowed participants to engage at their leisure in the creation of a portable personal alter, with objects that represented the five elements. While the artist himself was not able to attend the symposium, he provided his protocol for portable alters with guide sheets and recommendations for materials, which was very much appreciated. The Endicott College Art Therapy students facilitated the portable altar experience as seen in Figure 4. Participants were able to choose a small tin container into which they could add objects corresponding with the five elements, along with strips of paper that detailed hand drawn symbols, written quotes, and meditations. Objects included things like local sea glass and sea shells to represent water, feathers to represent air, stick matches and candles to represent fire, small twigs, and metal charms. One of the beauties of the portable alter is that the objects are not fixed in the container, they are able to move freely, which offered a meaningful takeaway for each participant to engage with and contemplate on even after the symposium.

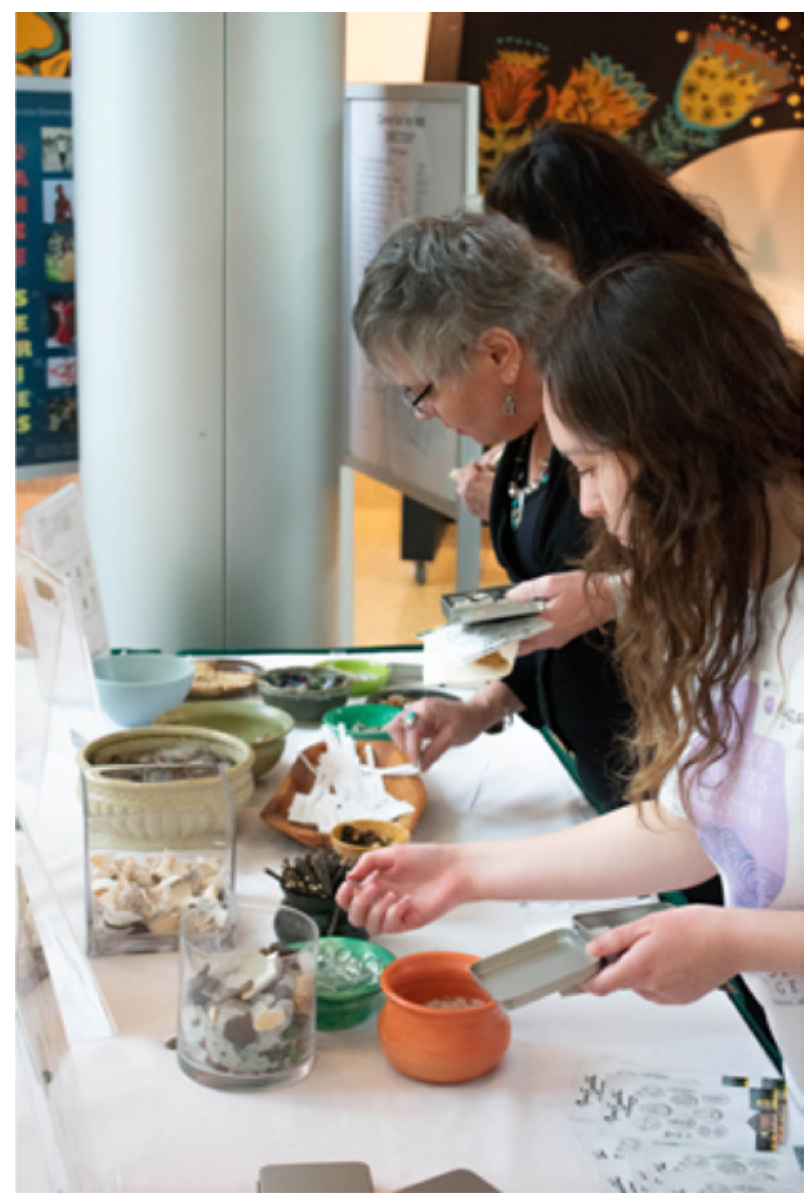

FIGURE 4 | Portable altars. 
Finally, while participants engaged with the open art activities there was a guqin performance on the small stage of the atrium. Shin Yi Yang, a native of Taiwan, and director of the Boston Gu Zheng ensemble and Boston Qin Society performed on the guqin. Yang wore traditional performance attire, with a traditional Chinese silk jacket, in order to perform on the ancient Chinese musical instrument. The guqin is a sevenstring zither-like instrument made of wood with a gentle and soft tone. According to Yang's website (2016) every Chinese scholar, throughout history, must learn four art forms, these include "qin (qin music), qi (chess), shu (calligraphy), and hua (painting)." With the qin being at the forefront it has much meaning in its history and power, specific to Confucianism and Taoism (para. 2). When Yang performed at the symposium, all of its participant paused, tuned in, and appreciated the powerful yet gentle Zen quality of the guqin. At the conclusion of her performance there was a feeling of love and exaltation in the atrium. The gentle Zen sound demonstrated the power of stillness, the yin, versus the active yang of the open art activities that preceded the environment of the atrium.

\section{Large Group Activities}

The individualized freedom of the morning activities deepened participants' understanding of the relevant polarities of yin-yang, as everyone joined together as a large group for the afternoon events. To reunite, all participants met in the Helftler Gallery for a 45 -minute talk by the artist Chunhong Wang. Wang's talk "Ink Painting as the Topographic Map of the Mind", displayed black ink paintings on white rice paper scrolls created by children with autism and cognitive impairments. The children/artists had worked with Wang in Beijing, China through her method of dimensional art dance therapy. In addition to the rice paper scrolls hung on the walls, pedestals displayed Chinese paper fans decorated with ink and natural tea to add rich coloration in yellow, red, and brown. Each ink painting metaphorically told a story of how the child created the work of art. The scroll paintings on the walls were made with a single movement of ink on paper using a large Chinese paint brush, while the ink work on the fans utilized smaller paint brushes and displayed more detailed Chinese characters and symbols. Wang informed the participants that the children's movements become visual with the representation of the ink, and how the child's movement allowed for deeper understanding of their own personal emotions, thoughts, and feelings. Wang's interactive lecture invited a robust discussion of how the black ink represents a response of the movement of energy in the body, through singing and movement activities, improved flow of energy, or ch'i had been brought forth in the children.

At the conclusion of the gallery talk, all participants returned to the Rose Theater where the initial morning talks took place. Participants were met with Daoist monk and tai chi master Shifu Zhou Xuan Yin, and acupuncturist Dr. Henry Lee for a 75-minute presentation on "Daoism and Traditional Chinese Medicine". Shifu explained that he grew up in a temple on Wudang Mountain where he was a student of Taiji Quan and Kung Fu. Trained in ritual arts, chanting, divination, and internal 
alchemy, he has dedicated his life to passing on the traditional Daoism arts. Lee has studied with Shifu for a number of years and has become a master student in his discipline. Shifu wore a long traditional black jacket, white leggings, and his hair in a top knot. He explained that not cutting his hair shows respect for the body's natural process, and that the top knot is unique to Daoism in that it serves as a continuation of energy from the body to the sky. During the lecture, Shifu spoke primarily in Chinese while Lee translated to English. Through a series of slides Shifu explained the relationships between yin and yang, chi, and the five-element theory. He identified Daoism as the treasure-house of Chinese culture as it is a belief and therefore a way of life. Shifu explained the use of swords by Daoist priests, and that the sword has spiritual properties designated by the elements it is made from. Finally, Shifu Zhou shared the story of the Japanese calligrapher and a samurai that challenged the other to a duel. Each day the calligrapher practiced his strokes with the paint brush. When it was time for the duel, the samurai watched the calligrapher draw his sword with grace and beauty, which caused the samurai to drop to his knees. "Whether the sword is in the martial artist's hand or the paintbrush in the calligrapher's; both movements flows with intention" (Xuan Yin, 2019). With the story at its conclusion Shifu and Lee invited their audience to the stage for a 30-minute practice of traditional tai chi exercise. As the attendees staggered their bodies across the stage in the theater Shifu came to the front to instruct the practice (see Figure 5).

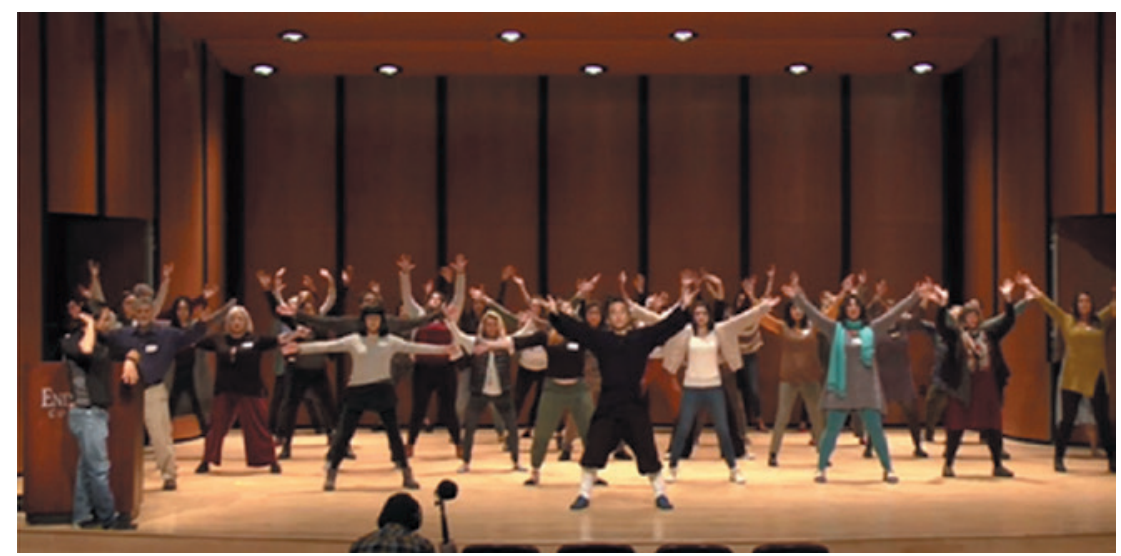

FIGURE 5 | Tai chi practice.

Audience members reportedly found the tai chi visually stunning to watch and participants said the movement felt invigorating, integrative, and that it was a meaningful way to wind down from the day. As the movement ended, Wang, Demaine, and Shifu joined together to facilitate closing the symposium day. While the group converged into a large circle on the stage, Demaine remarked on the way that the symposium integrated the two cultures, and brought forth a discussion that allowed for 
respect, compassion, and creativity. Shifu noted the calm and healing energy among the entire room and the creative beauty that was cultivated across the entire day. Wang then led the group in a movement-based closing that allowed each person in the room to independently and silently express one movement as they wished, as method of gratitude and farewell.

\section{Outcomes and Reflections}

The first bi-annual Endicott College Expressive Therapies symposium afforded a rich and diverse day of activities with its theme of Traditional Chinese Medicine, Arts, and Culture. The mission to support diversity within the expressive arts communities seemed to be achieved by the diverse voices of the presenters within their multi-arts disciplines. The expressive arts involve multiple art forms and with the presentations afforded by Chunhong Wang through Chinese paper cut, the traditional Chinese music performance by Shin Yi Yang, and tai chi with Shifu Zhou Xuan Yin and Lee, attendees were greeted with multiple forms of engagement and stimulation.

The daylong event allowed students and professionals to integrate a conversation that was waiting to happen. It is deeply profound how much humans can learn from one another within a culture of diversity. The arts are intrinsically grounded in cross-cultural experiences, and without acknowledging, exploring, and sharing our own methods, knowledge, and roots, we will not be able to fully practice to the best of our potential. In this author's opinion more forums are needed where individuals from different perspectives and areas of thinking, whether trained in traditional academic settings or through indigenous practices, from temples in the mountains to great performance halls, as we all have something to teach and to learn from one another. When it comes to the arts in healing, there are no limits so long as there is mutual respect, an open heart, and meaningful conversation.

The symposium brought together voices from across the globe. The day brought energy to the Endicott College campus through the participation of the symposium speakers and its attendees. Endicott College looks forward to the continuation of a bi-annual expressive therapies symposium with a dynamic and engaging new topic, one that encourages growth, support, diversity, and to nourish practitioners, students, and supporters within the expressive therapies community.

\section{Acknowledgments}

The author would like to give special thanks to Dean Mark Towner, Endicott College School of Visual and Performing Arts, and all of the Expressive Therapies Symposium presenters, as well as the International Expressive Arts Therapy Association, especially Dr. Youself Alajarma, Topaz Weiss, and Jennie Kristel.

\section{About the Author}

Krystal Demaine, PhD, REAT, MT-BC, RYT, is a board-certified music therapist, registered expressive arts therapist, certified yoga teacher, researcher, musician, artist, 
and mother. She serves as Associate Professor of Expressive Therapies at Endicott College in Massachusetts and is the founder of the Endicott College Expressive Therapies Symposium, bringing together collaborative voices on therapeutic healing perspectives from across the globe. Krystal's 20+ years as a therapist working with neurodiverse populations, wellness models, integrative medicine, grief support, and mental health have been published as book chapters and in peer reviewed articles. Her integrative arts and wellness practice with the heartbeat rhythm has provided the foundation to her life's work exploring The Roots and Rhythm of the Heart. www.krystaldemaine.com, Email: kdemaine@endciott.edu.

\section{References}

Demaine, K. (2015). Musical roots for healing: The five tone system in traditional Chinese medicine. In S. L. Brooke (Ed.), Therapists Creating a Cultural Tapestry: Using the Creative Therapies Across Cultures. (pp. 154-169). Springfield, IL: Charles C. Thomas.

Demaine, K. (2016). A reunion of east and west: Reflections on the roots of creative arts therapy and traditional Chinese medicine. Creative Arts in Education and Therapy: East and West, 2(1), 29-39.

Demaine, K. \& Richardson, J. F. (2015). The arts and natural health: A merging of creative art therapies and traditional Chinese medicine. In S. L. Brooke (Ed.), Therapists Creating a Cultural Tapestry: Using the Creative Therapies Across Culture. (pp. 57-72). Springfield, IL: Charles C. Thomas.

Kossak, M. (2015). Attunement in Expressive Arts Therapy: Toward an Understanding of Embodied Empathy. Springfield, IL: Charles C. Thomas.

Reid, D. (1996). The Shambhala Guide To Traditional Chinese Medicine: An Essential Introduction To The Theory And Practice Of An Ancient Healing Art. Boston, MA: Shambhala.

Yang, S. Y. (2016). Qin. Retrieved:// https://www.shinyiyang.com/qin. 